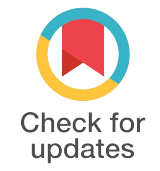

DOI: $10.29252 / \mathrm{ijcp}-26716$

\section{Prevalence of Non-Thromboembolic Incidental Findings on Computed Tomography-Pulmonary Angiography for Pulmonary Embolism}

\author{
Rama Bozorgmehr ', Mehdi Pishgahi 2,*, Pegah Mohaghegh ${ }^{3}$, \\ Morteza Saneitaheri ${ }^{2}$, Marziye Bayat ${ }^{2}$, Parastou Khodadadi ${ }^{2}$, \\ Ahmadreza Ghafori ${ }^{2}$ \\ ${ }^{1}$ Clinical Research Development Unit, Shohadaye Tajrish Hospital, Shahid \\ Beheshti University of Medical Sciences, Tehran, Iran \\ ${ }^{2}$ Shohadaye Tajrish Hospital, Shahid Beheshti University of Medical Sciences, \\ Tehran, Iran \\ ${ }^{3}$ Department of Community and Preventive Medicine, School of Medicine. Arak \\ University of Medical Sciences, Arak, Iran \\ * Corresponding author: Mehdi Pishghahi, Shohadaye Tajrish Hospital, Shahid
}

\begin{abstract}
Introduction: Computed Tomography-Pulmonary Angiography (CTPA) is a noninvasive imaging modality for direct diagnosis of pulmonary thromboembolism. The potential advantage of CTPA is possible alternative and incidental findings in cases that PTE is ruled out. This study was performed to determine the prevalence of incidental findings in CTPA in patients suspected to have PTE.

Methods: This cross-sectional retrospective study was performed in patients with suspected PTE admitted to Shohada-e-Tajish Hospital in 2014 and 2015 and underwent CTPA for final diagnosis. Incidental findings in CTPA and associated clinical symptoms were assessed.

Results: According to CTPA performed in 188 patients, PTE was diagnosed in 61 cases (32.4\%). Prevalence of incidental abnormal findings in the two groups with and without PTE were $93.7 \%$ and $90.9 \%$, respectively. The most common incidental finding was pleural effusion (42\%). There was no significant association between clinical symptoms and incidental findings in CTPA in patients with suspected pulmonary embolism ( $\mathrm{P}>$ $0.05)$. The only significant finding was association between lung mass and tachypnea $(\mathrm{P}=0.007)$.

Conclusion: In patients with primary clinical symptoms of suspected pulmonary embolism, in most cases there was a wide range of incidental findings and simultaneous pathologies in CTPA mimicking the primary symptoms of pulmonary embolism. However, in this study there was no significant association between clinical symptoms and incidental findings. Determination of definite indications of CTPA in patients with suspected pulmonary embolism is necessary.
\end{abstract}

\title{
INTRODUCTION
}

Annual prevalence rate of pulmonary thromboembolism (PTE) is 100-200 per 100000 populations [1] and monthly mortality rate ranges from $6.7 \%$ to $11 \%[2,3]$ and up to $30 \%$ in non-treated cases [4]. But autopsy studies demonstrate that these rates are lower than actual burden [5]. Under-diagnosis of PTE is due to wide range of changes in clinical presentations of PTE and also non-specific findings. Clinical findings of
PTE include dyspnea, chest pain, hemoptysis and tachypnea. In acute conditions with further alterations in pulmonary circulation or positive history of cardiopulmonary diseases, an unstable hemodynamic status including hypotension, low cardiac output symptoms and hypoxemia may be found [6]. Clinically suspected cases of PTE should be assessed by different tests according to clinical situations. Use of computed 
tomography pulmonary angiography (CTPA) has been increased in the recent years and it is used as an important diagnostic tool in cases with suspicion of PTE $[7,8]$. CTPA is a noninvasive imaging modality for direct diagnosis of intra-arterial pulmonary thrombosis and the results are accessible during 24 hours. The sensitivity of CTPA ranges from 64 to $100 \%$ with a specificity from 89 to $100 \%$ [9-11]. The CTPA results are positive for PTE in 6.6-60\% depending on the used PTE criteria [12-15]. Potential advantage of CTPA is possible alternative and incidental findings in cases that PTE is ruled out and other diagnoses can explain the clinical symptoms that may be treated appropriately. Differential diagnoses include pneumonia, cancer, pleural effusion, heart failure and Chronic Obstructive Pulmonary Disease (COPD) exacerbation. Also incidental findings include benign nodules, adenopathy and granulomatosis in $25.4 \%$ to $70 \%$ of CTPA results $[12,14]$. On the other hand, excess non-indicated use of CTPA is matter of debate due to intravascular contrast use, radiation exposure and costs [16]. The main aim of this study was determination of incidental findings (other than PTE) in CTPA in patients suspected to have PTE. Also the associations with clinical findings were assessed.

\section{METHODS}

This cross-sectional study was a portion of research project of "The association between the risk factors and clinical and para-clinical findings in patients suspected to have pulmonary thromboembolism". Regarding multiple variables and to reduce elongation of the matter, incidental findings were reported separately here. In this study, 188 patients suspected to have PTE admitted in Shohada-e-Tajrish Hospital in 2014 and 2015 who underwent CTPA were enrolled and assessed regarding incidental findings and the association with clinical findings.

The project was approved by the ethical committee and the required permissions from radiology ward was attained. All CTPA reports ordered by different parts of the hospital for patients with suspicion of PTE in 2014 and 2015 collected and data was extracted and recorded in checklist. Incidental findings in CTPA were divided into two categories of cardiac and non-cardiac findings.

\section{Statistics}

Data was described as mean and standard deviation for numerical variables and as frequency and percentage for categorical variables. Contingency coefficient was used to assess the association between categorical variables. The significance level was considered 0.05. Data analysis was performed by SPSS version 24.0 (SPSS Inc., Chicago, IL, USA).

\section{RESULTS}

In this study 188 patients including 85 males (45.2\%) and 103 females $(54.8 \%)$ with suspicion of pulmonary embolism underwent pulmonary CT scan. The mean age of subjects was $61.91 \pm 18.25$ years. In Table 1 , all CTPA findings in our patients are shown. The most common incidental findings were pleural effusion (42\%), atelectasis (26.1\%) and consolidation (24.5\%).

Table 1. Prevalence of Findings in Computed Tomography Pulmonary Angiography in Patients Suspected to Pulmonary Embolism

\begin{tabular}{lc}
\hline Incidental finding & Number (Frequency) \\
\hline Consolidation & $46(24.5 \%)$ \\
\hline Atelectasis & $49(26.1 \%)$ \\
\hline Pleural effusion & $79(42 \%)$ \\
\hline Pneumothorax & $6(3.2 \%)$ \\
\hline Nodule & $6(3.2 \%)$ \\
Mass & $6(3.2 \%)$ \\
\hline Lymphadenopathy & $20(10.6 \%)$ \\
\hline Cardiac findings & $12(6.4 \%)$ \\
\hline Miscellaneous & $46(24.5 \%)$ \\
\hline Emboli & $61(32.4 \%)$ \\
\hline
\end{tabular}

Incidental cardiac findings in this study included aortic calcification, cardiomegaly, coronary artery calcification and pericardial effusion. The related prevalence in CTPA in the two groups with and without pulmonary embolism was assessed and the results are shown in Table 2.

As demonstrated in Table 2, there was no statistically significant difference between incidental findings in the two groups with and without pulmonary embolism. Correlation between incidental radiological findings and clinical symptoms in all patients with suspected pulmonary embolism is shown in Table 3 . The only significantly different finding was the association of consolidation and tachypnea $(\mathrm{P}=0.007)$.

There was no significant association between clinical symptoms and CTPA findings in the two groups with and without embolism $(\mathrm{P}>0.05)$.

\section{DISCUSSION}

Total prevalence of pulmonary embolism in our 188 patients was $32.4 \%$. According to the literature, the prevalence of pulmonary embolism in CTPA in suspected cases of pulmonary embolism has been reported as $10 \%$ to $30 \%[8,15,17]$. The most common radiological findings requiring follow-up in patients with and without pulmonary embolism was pleural effusion (40.9\% versus $44.3 \%$ ).

According to the study by Richman et al. in patients with suspected pulmonary embolism CTPA would have evidences for a differential diagnosis in most cases [18]. According to the results of Kelly $\mathrm{W}$ et al., patients undergoing CTPA for assessment of probable pulmonary embolism have a range of symptoms and signs related to pulmonary embolism and other simultaneous pathologies. While these patients may have no pulmonary embolism, there are a wide range of incidental simultaneous pathologies to guide differential diagnoses [19]. 

Table 2. Comparison of Computed Tomography-Pulmonary Angiography Findings in Patients with and Without Pulmonary Thromboembolism

\begin{tabular}{lccc}
\hline & Emboli & Yes (N=61) & P-value \\
\hline Lymphadenopathy & No $\mathbf{N = 1 2 7})$ & $5(8.2 \%)$ & 0.4 \\
Mass (<3mm) & $15(11.8 \%)$ & $1(1.6 \%)$ & 0.6 \\
Pneumothorax & $5(3.9 \%)$ & $2(3.3 \%)$ & 1.0 \\
Atelectasis & $4(3.1 \%)$ & $12(19.7 \%)$ & 0.1 \\
Consolidation & $37(29.1 \%)$ & $15(24.6 \%)$ & 0.9 \\
\hline Pleural effusion & $31(24.4 \%)$ & $27(44.3 \%)$ & 0.6 \\
Nodule (>3mm) & $52(40.9 \%)$ & $0(0 \%)$ & 0.1 \\
\hline Others (miscellaneous) & $6(4.7 \%)$ & $15(24.6 \%)$ & 0.9 \\
Cardiac finding & $31(24.4 \%)$ & $2(3.3 \%)$ & 0.2 \\
\hline
\end{tabular}

Data in table are presented as No. (\%)

Table 3. Association of Incidental Findings in Computed Tomography-Pulmonary Angiography and Symptoms in all Suspected Patients to Pulmonary Embolism

\begin{tabular}{|c|c|c|c|c|c|}
\hline & Hemoptysis & tachypnea & tachycardia & chest pain & Outcome \\
\hline \multicolumn{6}{|l|}{ Nodule } \\
\hline Contingency coefficient & 0.019 & 0.035 & 0.052 & 0.063 & 0.039 \\
\hline $\mathrm{P}$-value & 0.7 & 0.630 & 0.4 & 0.385 & 0.5 \\
\hline \multicolumn{6}{|l|}{ Mass } \\
\hline Contingency coefficient & 0.019 & 0.063 & 0.052 & 0.063 & 0.039 \\
\hline $\mathrm{P}$-value & 0.7 & 0.389 & 0.4 & 0.385 & 0.5 \\
\hline \multicolumn{6}{|l|}{ Pneumothorax } \\
\hline Contingency coefficient & 0.019 & 0.035 & 0.063 & 0.052 & 0.099 \\
\hline $\mathrm{P}$-value & 0.7 & 0.630 & 0.3 & 0.479 & 0.1 \\
\hline \multicolumn{6}{|l|}{ Pleural effusion } \\
\hline Contingency coefficient & 0.016 & 0.019 & 0.038 & 0.038 & 0.014 \\
\hline $\mathrm{P}$-value & 0.8 & 0.792 & 0.6 & 0.607 & 0.8 \\
\hline \multicolumn{6}{|l|}{ consolidation } \\
\hline Contingency coefficient & 0.061 & 0.194 & 0.115 & 0.021 & 0.057 \\
\hline $\mathrm{P}$-value & 0.4 & 0.007 & 0.1 & 0.775 & 0.4 \\
\hline \multicolumn{6}{|l|}{ Atelectasis } \\
\hline Contingency coefficient & 0.056 & 0.069 & 0.077 & 0.031 & 0.006 \\
\hline $\mathrm{P}$-value & 0.4 & 0.344 & 0.2 & 0.673 & 0.9 \\
\hline \multicolumn{6}{|l|}{ Lymphadenopathy } \\
\hline Contingency coefficient & 0.131 & 0.119 & 0.098 & 0.033 & 0.087 \\
\hline $\mathrm{P}$-value & 0.07 & 0.101 & 0.1 & 0.651 & 0.2 \\
\hline
\end{tabular}

According to the study by Pezeshki Rad et al. the most common incidental findings requiring follow up in CTPA in patients with suspected pulmonary embolism include pulmonary consolidation (33.2\%) and pleural effusion (48.7\%). In addition, CTPA may demonstrate pathological findings that would require follow-up. Hence it is important to diagnose and report these findings because some of them would change treatment and prognosis of patients suspected to have pulmonary embolism [20].

In our study the most common incidental findings in CTPA needing follow-up were pleural effusion, atelectasis and consolidation. Results of the study by Ozkin et al. in 2014 revealed that the most common pathological findings other than pulmonary embolism in CTPA were atelectasis (39.9\%) and ground glass (23.7\%) [17]. These results are also in congruence with Tresoldi S et al. and Perelas A et al. [14, 17, 21].
According to the study by William B et al. in 33\% of CTPA reports there were some findings suggesting other differential diagnoses and $24 \%$ had incidental findings needing clinical follow-up [22]. In our study in $6.3 \%$ of cases with PTE and $9.1 \%$ of those without PTE there was no incidental finding in CTPA, which is in congruence with studies by Perales A et al. and Ozakin E et al. who reported normal CTPA in $12.5 \%$ and $29.3 \%$ cases, respectively $[8,14,17]$.

According to Ferreira E et al., CTPA is beneficial for final diagnosis of suspected cases of pulmonary embolism, because it would certify the diagnosis and help to recognize incidental findings that are useful for differential diagnoses in most cases with clinical symptoms related to suspected pulmonary embolism [23]. Contrary to our study, Hall, William B et al. showed that many of these findings could be also found in high-quality chest $\mathrm{X}$ ray. Hence for prevention of 
unnecessary CTPA, determination of definite indications in patients with suspected symptoms of pulmonary embolism is mandatory, especially in emergency wards [22]. Therefore, emergency specialists and general physicians working in emergency ward should be familiar with approaches recommended for assessment of lung nodules and other similar pulmonary findings, because use of CTPA would reveal many unimportant incidental findings.

Currently some researchers have hypothesized that routine use of algorithms and guidelines for the diagnosis of pulmonary emboli may increase the number of true positive cases of PTE and CTPA $[6,24]$. Hence differences in the rate of CTPA use and number of diagnosed PTE cases by this method may be affected by utilization rate of algorithms by physicians. Therefore, use of these guidelines to have single standard approach in suspected cases of pulmonary embolism is necessary.

\section{CONCLUSION}

In patients with suspected pulmonary embolism, a wide range of incidental findings and simultaneous pathologies could be found in CTPA. Therefore, the use of clinical algorithms is necessary for more definite approach to differential diagnoses of pulmonary embolism.

\section{Conflict of Interest}

There is no conflict of interest in this study

\section{REFERENCES}

1. Heit JA. The epidemiology of venous thromboembolism in the community. Arterioscler Thromb Vasc Biol. 2008;28(3):3702. doi: 10.1161/ATVBAHA.108.162545 pmid: 18296591

2. Aujesky D, Obrosky DS, Stone RA, Auble TE, Perrier A, Cornuz J, et al. A prediction rule to identify low-risk patients with pulmonary embolism. Arch Intern Med. 2006;166(2):169-75. doi: 10.1001/archinte.166.2.169 pmid: 16432084

3. Casazza F, Becattini C, Bongarzoni A, Cuccia C, Roncon L, Favretto G, et al. Clinical features and short term outcomes of patients with acute pulmonary embolism. The Italian Pulmonary Embolism Registry (IPER). Thromb Res. 2012;130(6):847-52. doi: 10.1016/j.thromres.2012.08.292 pmid: 22921592

4. Kim KI, Muller NL, Mayo JR. Clinically suspected pulmonary embolism: utility of spiral CT. Radiology. 1999;210(3):693-7. doi: 10.1148/radiology.210.3.r99mr01693 pmid: 10207469

5. Torbicki A, Perrier A, Konstantinides S, Agnelli G, Galie N, Pruszczyk P, et al. Guidelines on the diagnosis and management of acute pulmonary embolism: the Task Force for the Diagnosis and Management of Acute Pulmonary Embolism of the European Society of Cardiology (ESC). Eur Heart J. 2008;29(18):2276-315. doi: 10.1093/eurheartj/ehn310 pmid: 18757870

6. Members ATF, Konstantinides SV, Torbicki A, Agnelli G, Danchin N, Fitzmaurice D, et al. 2014 ESC Guidelines on the diagnosis and management of acute pulmonary embolism: The Task Force for the Diagnosis and Management of Acute Pulmonary Embolism of the European Society of Cardiology (ESC) Endorsed by the European Respiratory Society (ERS). Eur Heart J. 2014;35(43):3033-80.

7. Chandra S, Sarkar PK, Chandra D, Ginsberg NE, Cohen RI. Finding an alternative diagnosis does not justify increased use of CT-pulmonary angiography. BMC Pulm Med. 2013;13:9. doi: 10.1186/1471-2466-13-9 pmid: 23388541

8. van Strijen MJ, de Monye W, Schiereck J, Kieft GJ, Prins MH, Huisman MV, et al. Single-detector helical computed tomography as the primary diagnostic test in suspected pulmonary embolism: a multicenter clinical management study of 510 patients. Ann Intern Med. 2003;138(4):307-14. doi: $10.7326 / 0003-4819-138-4-200302180-00009$ pmid: 12585828

9. de Monye W, Pattynama PM. Contrast-enhanced spiral computed tomography of the pulmonary arteries: an overview. Semin Thromb Hemost. 2001;27(1):33-9. doi: 10.1055/s2001-12845 pmid: 11288946

10. Mullins MD, Becker DM, Hagspiel KD, Philbrick JT. The role of spiral volumetric computed tomography in the diagnosis of pulmonary embolism. Arch Intern Med. 2000;160(3):293-8. doi: $10.1001 /$ archinte.160.3.293 pmid: 10668830

11. Rathbun SW, Raskob GE, Whitsett TL. Sensitivity and specificity of helical computed tomography in the diagnosis of pulmonary embolism: a systematic review. Ann Intern Med. 2000;132(3):227-32. doi: 10.7326/0003-4819-132-3200002010-00009 pmid: 10651604

12. Deonarine $\mathrm{P}$, de Wet C, McGhee A. Computed tomographic pulmonary angiography and pulmonary embolism: predictive value of a d-dimer assay. BMC Res Notes. 2012;5:104. doi: 10.1186/1756-0500-5-104 pmid: 22340133

13. Crichlow A, Cuker A, Mills AM. Overuse of computed tomography pulmonary angiography in the evaluation of patients with suspected pulmonary embolism in the emergency department. Acad Emerg Med. 2012;19(11):1219-26. doi: 10.1111/acem. 12012 pmid: 23167851

14. Perelas A, Dimou A, Saenz A, Rhee JH, Teerapuncharoen K, Rowden A, et al. Incidental findings on computed tomography angiography in patients evaluated for pulmonary embolism. Ann Am Thorac Soc. 2015;12(5):689-95. doi: 10.1513/AnnalsATS.201404-144OC pmid: 25713998

15. van Es J, Douma RA, Schreuder SM, Middeldorp S, Kamphuisen PW, Gerdes VEA, et al. Clinical impact of findings supporting an alternative diagnosis on CT pulmonary angiography in patients with suspected pulmonary embolism. Chest. 2013;144(6):1893-9. doi: 10.1378/chest.13-0157 pmid: 23989896

16. Schattner A. Computed tomographic pulmonary angiography to diagnose acute pulmonary embolism: the good, the bad, and the ugly: comment on "The prevalence of clinically relevant incidental findings on chest computed tomographic angiograms ordered to diagnose pulmonary embolism". Arch Intern Med. 2009;169(21):1966-8. doi: 10.1001/archinternmed.2009.400 pmid: 19933957

17. Ozakin E, Kaya FB, Acar N, Cevik AA. An analysis of patients that underwent computed tomography pulmonary angiography with the prediagnosis of pulmonary embolism in the emergency department. ScientificWorldJournal. 2014;2014:470358. doi: 10.1155/2014/470358 pmid: 24955406

18. Richman PB, Courtney DM, Friese J, Matthews J, Field A, Petri $\mathrm{R}$, et al. Prevalence and significance of nonthromboembolic findings on chest computed tomography angiography performed to rule out pulmonary embolism: a multicenter study of 1,025 emergency department patients. Acad Emerg Med. 2004;11(6):642-7. pmid: 15175202

19. Kelly WC, Lynn SB. PE or no PE? Alternative diagnoses. Appl Radiol. 2018.

20. Pezeshki Rad M, Farrokh Tehrani D, Reihani H, Faghih Sabzevari SH, Rajabi M. Incidental findings in patients evaluated for pulmonary embolism using computed tomography angiography. J Cardiol-Thorac Med. 2014;2(2):162-6.

21. Tresoldi S, Kim YH, Baker SP, Kandarpa K. MDCT of 220 consecutive patients with suspected acute pulmonary embolism: incidence of pulmonary embolism and of other acute or non-acute thoracic findings. Radiol Med. 
2008;113(3):373-84. doi: 10.1007/s11547-008-0262-9 pmid: 18493774

22. Hall WB, Truitt SG, Scheunemann LP, Shah SA, River MP, Parker LA, et al. The prevalence of clinically relevant incidental findings on chest computed tomographic angiograms ordered to diagnose pulmonary embolism. Arch Intern Med. 2009;169(21):1961-5. doi: 10.1001 /archinternmed.2009.360 pmid: 19933956
23. Ferreira EV, Gazzana MB, Sarmento MB, Guazzelli PA, Hoffmeister MC, Guerra VA, et al. Alternative diagnoses based on CT angiography of the chest in patients with suspected pulmonary thromboembolism. J Bras Pneumol. 2016;42(1):35-41. doi: 10.1590/S1806-37562016000000105 pmid: 26982039

24. Yamamoto T. Management of patients with high-risk pulmonary embolism: a narrative review. J Intensive Care. 2018;6:16. doi: 10.1186/s40560-018-0286-8 pmid: 29511564 